\title{
Parametric Design of Involute spur gear transmission system based on Pro/Engineer and MathCAD
}

\author{
Zongzheng $\mathrm{Hu}^{1, \mathrm{a}}, \mathrm{Bin} \mathrm{Xu}^{2, \mathrm{~b}}$ \\ ${ }^{1}$ Lanzhou Vocational Technical college. Lanzhou. 730070. China \\ ${ }^{2}$ Shenyang Institute of Technology. Fushun. 113122. China \\ ahuzongzheng_Izy@163.com, bxubin_0907@126.com
}

\begin{abstract}
Keywords: Pro/E; Behavioral-characteristic modeling; Parametric design; Optimal design; MathCAD Abstract. It's usually a complex computing process of mechanical transmission components design. In this paper, a flexible and efficient method has been adopted based on the behavioral-characteristic modeling technique of Pro/E software and the powerful computing function of MathCAD software to optimize the gear drive system design. This method proposes a flexible and efficient method to solve the actual engineering problem, it also provides a train of thought for automation and intelligent design.
\end{abstract}

\section{Introduction}

With the continuous development of CAD/CAM/CAE technology, the parametric design modeling technology has been widely applied in our life. The parametric design procedures is action that generate parametric models where geometrical components are consider as variables. Using this method, a series of similar shape models can be redesigned precisely and efficiently. As a typical parametric design software, Pro/E's feature modeling technology can modify model characteristic parameters automatically so as to achieve the goal of design process. This paper offers a new conceptual framework of parametric design for involute spur gears on the basis of the behavioral-characteristic modeling technique of Pro/E software and the powerful computing function of MathCAD software.

\section{Gear parametric modeling}

modeling approaches. Gear design involves many geometric parameters. For standard gear, when basic parameters $(z, m, \alpha) h_{a}{ }^{*} c^{*}$ are determined, its tooth profile and dimensions are completely sure. Bring in tooth shape parameters to control tooth shape, establish the connection between the original parameters and the independent parameters with relationship tool, generate involute with curve equation. build spur gear entity model accurately through "stretch" and "array" feature modeling. Detailed steps, please refer to the related literature.

optimization design ideas. Due to the advantages of easy fabrication and low cost, soft tooth surface gear transmission is widely used in the cases of transmission requirements for accuracy, strength and speed is not high; transmission power is low or gear size requirements is low. Considered the performance requirements and working conditions of soft tooth surface gear, get the gear basic parameters of the initial value through Minimize function of MathCAD with the minimum gear center distance as the goal, to determine all the geometric parameters of gear system.

\section{The mathematical model of parameter optimization}

The objective function and design variables. Set minimum gear transmission center distance as the optimization target, the target function can be written as:

$$
f(x)=\frac{m_{1} z_{1}}{2}\left(1+i_{1}\right)+\frac{m_{2} z_{3}}{2}\left(1+\frac{i}{i_{1}}\right)
$$


$\mathrm{m}_{1}$-the modulus of high-speed gear;

$\mathrm{m}_{2}$-the modulus of low-speed gear;

$\mathrm{z}_{1}$-the number of teeth on the high-speed gear;

$\mathrm{z}_{2}$ - the number of teeth on the low-speed gear;

$\mathrm{i}_{1}$ - high-speed transmission ratio;

i-total transmission ratio.

As formula (1) shows, the total center distance of gear transmission is related to gear numbers, module, the first level ratio and coefficient of facewidth. so choose these parameters as independent design variables.

$$
x=\left(m_{1}, m_{2}, z_{1}, z_{3}, \phi_{d 1}, \phi_{d 2}, i_{1}\right)=\left(x_{1}, x_{2}, x_{3}, x_{4}, x_{5}, x_{6}, x_{7}\right)
$$

Constraints analysis. tooth surface contact strength constraint conditions. Contact stress is cyclic alternating stress, constraints of tooth surface contact strength is as follow:

$$
f(x)_{1}=\left[\sigma_{H}\right]_{1}-Z_{H} Z_{E} \sqrt{\frac{2 K_{1} T_{1}}{\phi_{d 1} d_{1}^{3}} \bullet \frac{i_{1}+1}{i_{1}}} \geq 0
$$

$K_{1}=K_{A 1} K_{V 1} K_{\alpha 1} K_{\beta 1}, d_{1}=m_{1} z_{1},\left[\sigma_{H}\right]$

$\left[\sigma_{H}\right]$-allowable contact stress;

$Z_{H}$ - node area coefficient;

$Z_{E}$-materials elasticity modulus;

$K_{A}$-using coefficient

$K_{V}$-Dynamic load coefficient;

$K_{\alpha}$ - load distribution coefficient between teeth;

$K_{\beta}$-Tooth direction load distribution coefficient;

$T_{1}$-torque of High speed transmission;

$$
\left.f(x)_{2}=\left[\sigma_{H}\right]_{2}-Z_{H} Z_{E} \sqrt{\frac{2 K_{2} T_{2}}{\phi_{d 2} d_{2}{ }^{3}} \bullet\left(1+\frac{i_{1}}{i}\right.}\right) \geq 0
$$

$K_{2}=K_{A 2} K_{V 2} K_{\alpha 2} K_{\beta 2} \quad d_{2}=m_{2} z_{2}$

$T_{2}$ - torque of low speed transmission;

\section{The tooth root bending strength constraint conditions.}

$$
\begin{aligned}
& f(x)_{3}=\left[\sigma_{F}\right]_{1}-\frac{2 K_{1} T_{1}}{\phi_{d 1} m_{1}{ }^{3} z_{1}{ }^{2}} Y_{F a 1} Y_{S a 1} \geq 0 \\
& f(x)_{4}=\left[\sigma_{F}\right]_{2}-\frac{2 K_{2} T_{2}}{\phi_{d 2} m_{2}{ }^{3} z_{3}{ }^{2}} Y_{F a 3} Y_{S a 3} \geq 0
\end{aligned}
$$

$\left[\sigma_{F}\right]$-Allowable tooth root bending strength ;

$Y_{F a}$-Tooth shape coefficient ;

$Y_{S a}$-Stress correction coefficient.

Modulus constraint. To the power transmission gear, the average modulus is greater than $2 \mathrm{~mm}$, constraints can be expressed as follows:

$$
\begin{aligned}
& f(x)_{5}=m_{1}-2 \geq 0 \\
& f(x)_{6}=m_{2}-2 \geq 0
\end{aligned}
$$

For the pressure Angle of $20^{\circ}$ standard spur gears, in order to avoid the root cutting, teeth should be greater than 17,so: 


$$
\begin{aligned}
& f(x)_{7}=z_{1}-17 \geq 0 \\
& f(x)_{8}=z_{3}-17 \geq 0 \quad(9)
\end{aligned}
$$

The choice of coefficient of tooth width.For standard spur gear transmission, normally require: $0.8 \leq \phi_{d} \leq 1.4$,so:

$$
\begin{aligned}
& f(x)_{9}=\phi_{d 1}-0.8 \geq 0 \\
& f(x)_{10}=1.4-\phi_{d 1} \geq 0 \\
& f(x)_{11}=\phi_{d 2}-0.8 \geq 0 \\
& f(x)_{12}=1.4-\phi_{d 2} \geq 0
\end{aligned}
$$

Optimization design based on MathCAD. By calling two functions of MathCAD : "maximize" and "minimize", the maximum and minimum value of Objective function can be obtained. Work steps are shown in figure 1:

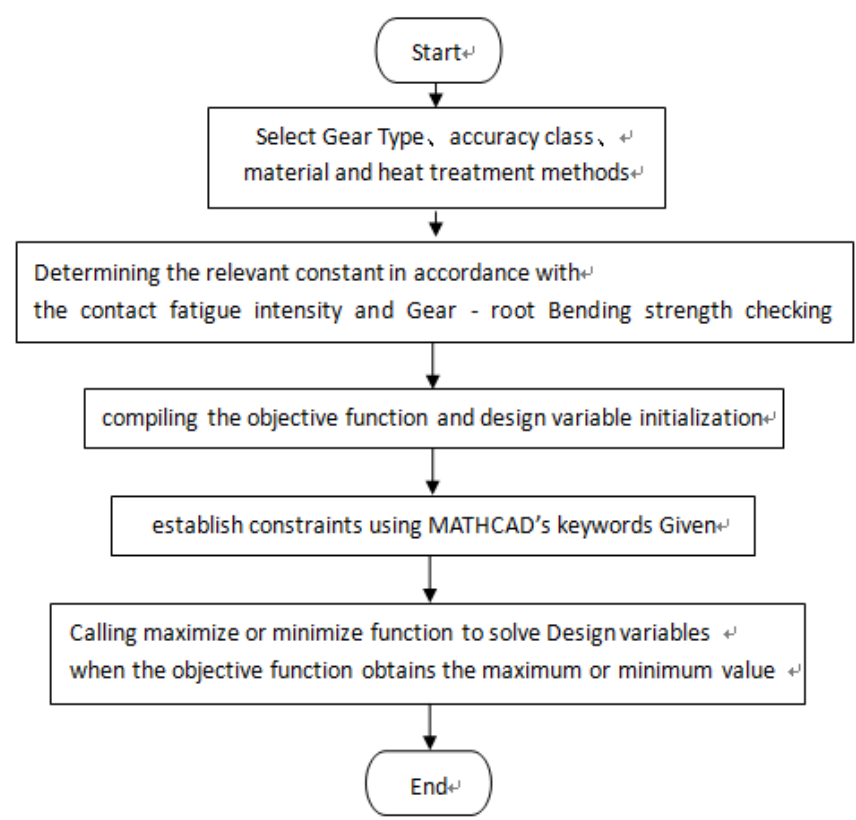

Fig.1 Optimization design flow chart

MathCAD optimization analysis. MathCAD is an engineering calculation software exploited by PTC company which is widely used in the engineering field of data calculating, graphics and text processing. Its powerful ability of analysis and calculation can well make up the calculation disadvantages of Pro/E. The MathCAD analysis flow chart is shown in figure 2.

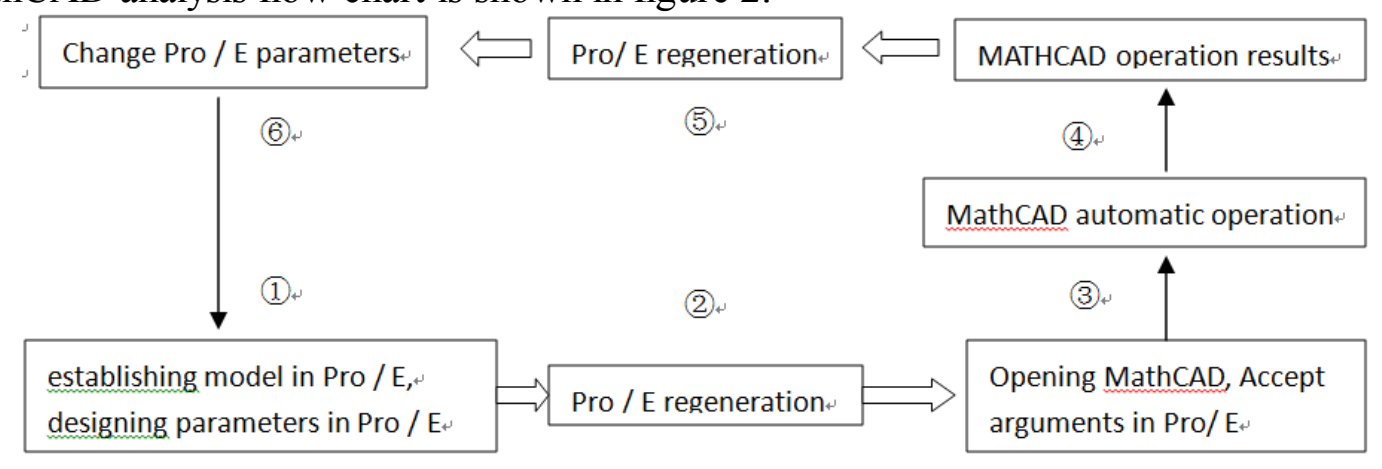

Fig. 2 MathCAD analysis workflow

Given that a secondary expansion speed reducer is under design. Known high-speed shaft power input $\mathrm{P} 1=6.2 \mathrm{~kW}$, high-speed spindle speed $\mathrm{n} 1=960 \mathrm{r} / \mathrm{min}$, the total transmission ratio $\mathrm{I}=15$, driven by a motor, the working life is 15 years (set work 300 days per year). The Material of pinion is steel 45 
\#, quenched-tempered HB228 255. Belt conveyor requirements work smoothly. Request to determine the overall scheme of the main parameters of gears according to the minimum centre distance.Create a Pro/E assembly file, assemble level 2 gear with any center distance, the assembly constraints type is pin. create a new parameter $Z D=1$ in Pro/E transmitted to MathCAD for calculation.

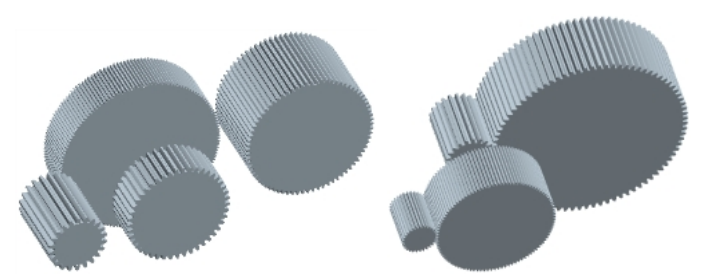

( a ) The initial assembly model ( b ) The updated model

Fig. 3 Drive gear assembly

Click the main menu "analysis" $\rightarrow$ "external analysis" $\rightarrow$ "MathCAD analysis".click "new document" button in the pop-up dialog box. Open the software MathCAD, create a New MathCAD document and optimization mathematical model is established. Calculate, and finally obtain the modulus, tooth number of each gear, dividing circle diameter, tooth width and other geometrical parameters.

$$
\begin{aligned}
& \quad f(x 1, x 2, x 3, x 4, x 5, x 6, x 7)=\frac{x 1 \cdot x 3}{2} \cdot(1+x 7)+\frac{x 2 \cdot x 4}{2} \cdot\left(1+\frac{15}{x 7}\right) \\
& \mathrm{x} 1:=2 x 2:=3 \times 3:=19 x 4:=19 x 5:=1 \times 6:=1 \times 7:=6.7 \\
& 475-2.5 \cdot 189.8 \cdot \sqrt{\frac{2 \cdot 1.908 \cdot 0.617 \cdot 10^{5}}{x 5 \cdot x 1^{3} \cdot x 3^{3}}}
\end{aligned}
$$

Known$$
\sqrt{\frac{x 7+1}{x 7}} \geq 0
$$$$
485-2.5 \cdot 189.8 \cdot \sqrt{\frac{2 \cdot 1.922 \cdot 0.617 \cdot 10^{5} \cdot 0.98 \cdot x 7}{x 6 \cdot x 2^{3} \cdot x 4^{3}}}
$$$$
\sqrt{1+\frac{x 7}{15}} \geq 0
$$$$
264-\frac{2 \cdot 1.716 \cdot 0.617 \cdot 10^{5}}{x 5 \cdot \times 3^{2} \cdot x 1^{3}} \cdot 2.16 \cdot 1.81 \geq 0
$$$$
267-\frac{2 \cdot 1.716 \cdot 0.617 \cdot 10^{5} \cdot 0.98 \cdot x 7}{x 6 \cdot x 4^{2} \cdot x 2^{3}} \cdot 2.2 \cdot 1.78 \geq 0
$$

$x 1-2 \geq 0$

$x 2-2 \geq 0$

$1.4-x 6 \geq 0$

$$
\text { Minimize }(f, x 1, x 2, x 3, x 4, x 5, x 6, x 7)=\left(\begin{array}{c}
2 \\
4.291 \\
29.97 \\
20.626 \\
1.4 \\
1.4 \\
3.531
\end{array}\right)
$$

Round off the data: $m m 1=2 m z 1=30 f d 1=1.4 m m 2=4 m z 3=20 f d 2=1.4 m i 1=3.5$ 
variables explanation. During the process of MathCAD analysis, in order to complete the data transfer between Pro/E and MathCAD, a lable is needed to be defined for the variable. Select the definition of a variable, click - "properties", then found in the "display" TAB "label" and enter "proe $2 \mathrm{mc}$ " or "mc2proe".In this case, the $\mathrm{Z} 1$ need to add the first label, $\mathrm{mm} 1 、 \mathrm{~mm} 2 、 \mathrm{mz} 1 、 \mathrm{mz} 3$ etc. need to return to the Pro/E to add a second label. After above operation, save the file as" gear.xmcd". Click the "load file" button, choose the new "gear. xmcd " file in the open file browser. Click "add parameter", Choose parameters which are set in Pro/E. choose corresponding parameters in MathCAD in "input to select" dialog box after click "ok". Input MathCAD variables into Pro/E, click the "output", select necessary parameters to facilitate subsequent calculations, such as size of gear modulus, tooth number, tooth width, etc. The parameters are shown in fig. 4 .

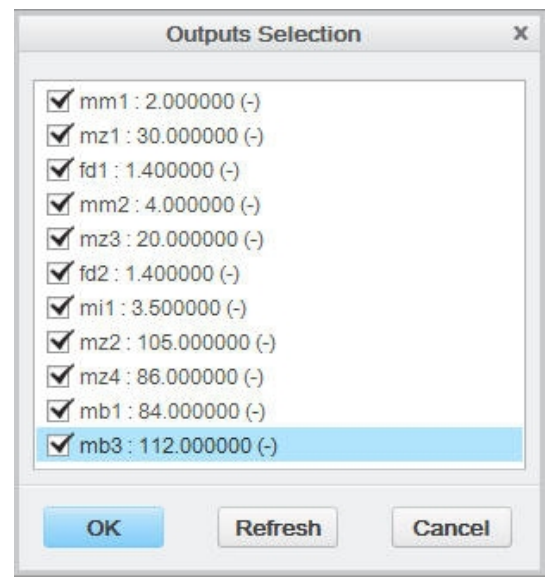

Fig.4 The output selection

Click "calculate", "add features" successively, analyze and name the file. Click "close", complete the analysis characteristics creation, result parameters is established automatically In this feature. Add" relationship" in Pro/E. To update the parameters values in Pro/E, need to add corresponding relation in Pro/E, such as:

$$
\begin{aligned}
& \text { M:2=MC_MM1:FID_ANALYSIS1 } \\
& \mathrm{Z}: 2=\mathrm{MC} \text { MZ1:FID_ANALYSIS1 } \\
& \text { B:2=MC_MB1:FID_ANALYSIS } 1 \\
& \text { Z:4=MC_MZ2:FID_ANALYSIS1 } \\
& \text { M:4=MC_MM1:FID_ANALYSIS1 } \\
& \mathrm{B}: 4=\mathrm{B}: 2+5 \\
& \mathrm{~d} 0=(\mathrm{M}: 2 * \mathrm{Z}: 2+\mathrm{M}: 4 * \mathrm{Z}: 4) / 2 \\
& \mathrm{Z}: 6=\text { MC_MZ3:FID_ANALYSIS } 1 \\
& \text { M:6=MC_MM2:FID_ANALYSIS1 } \\
& \text { B:6=MC_MB3:FID_ANALYSIS1 } \\
& \mathrm{Z}: 8=\text { MC_MZ4:FID_ANALYSIS } 1 \\
& \text { M:8=MC_MM2:FID_ANALYSIS1 }
\end{aligned}
$$

Modify the relevant parameters in Pro/E values, regeneration model, the calculated results in MathCAD also change dynamicly. The updating data in MathCAD transfer to Pro/E, the model can be regenerated again. Thus the data transmission, calculation and synchronous update between MathCAD and PRO/E is realized. The result is shown in fig.3(b).

\section{Conclusions}

Traditional multi-stage expansion design of gear transmission system, on the one hand, the computing is complex and error-prone; on the other hand, it can't optimize drivetrain outer contour size, volume, weight and the manufacturing cost. In this paper, by using Pro/E and MathCAD software 
integration solutions, achieved the minimum dimensions optimization design of gear transmission system, realized synchronous update of the optimization results and the entity model. Design method is efficient, intelligent, simple with certain practical value and popularization value.

\section{Reference:}

[1] Xinyi Jiang, Jingfeng Shen, Liangwei Zhong.Parametric Design of Batch Flender's Gear Units Based on Pro/Engineer. Modern Applied Science, 2011, Vol.5 (3).

[2] Xiaohe Deng, Lin Hua, Xinghui Han, Davide Spinello. Research on the Design and Modification of Asymmetric Spur Gear. Mathematical Problems in Engineering, 2015, Vol.2015.

[3] Xueyi Li, Shoubo Jiang, Qingliang Zeng. Optimization of Two-Stage Cylindrical Gear Reducer with Adaptive Boundary Constraints. Journal of Software, 2013, Vol.8 (8), pp.2052-2057. 